\title{
Automated Classification to Predict the Progression of Alzheimer's Disease Using Whole-Brain Volumetry and DTI
}

\author{
Won Beom Jung ${ }^{1}$, Young Min Lee ${ }^{2,3}$, Young Hoon $\mathrm{Kim}^{4}$, and Chi-Woong Mun ${ }^{1} \bowtie$ \\ 1Department of Biomedical Engineering/u-HARC, Inje University, Gimhae, Republic of Korea \\ ${ }^{2}$ Department of Psychiatry, Pusan National University Hospital, Busan, Republic of Korea \\ ${ }^{3}$ Medical Research Institute, Pusan National University Hospital, Busan, Republic of Korea \\ ${ }^{4}$ Department of Psychiatry, Medical School, Inje University, Haeundae Paik Hospital, Busan, Republic of Korea
}

Objective This study proposes an automated diagnostic method to classify patients with Alzheimer's disease (AD) of degenerative etiology using magnetic resonance imaging (MRI) markers.

Methods Twenty-seven patients with subjective memory impairment (SMI), 18 patients with mild cognitive impairment (MCI), and 27 patients with AD participated. MRI protocols included three dimensional brain structural imaging and diffusion tensor imaging to assess the cortical thickness, subcortical volume and white matter integrity. Recursive feature elimination based on support vector machine (SVM) was conducted to determine the most relevant features for classifying abnormal regions and imaging parameters, and then a factor analysis for the top-ranked factors was performed. Subjects were classified using nonlinear SVM.

Results Medial temporal regions in $\mathrm{AD}$ patients were dominantly detected with cortical thinning and volume atrophy compared with SMI and MCI patients. Damage to white matter integrity was also accredited with decreased fractional anisotropy and increased mean diffusivity (MD) across the three groups. The microscopic damage in the subcortical gray matter was reflected in increased MD. Classification accuracy between pairs of groups (SMI vs. MCI, MCI vs. AD, SMI vs. AD) and among all three groups were $84.4 \%( \pm 13.8$ ), $86.9 \%( \pm 10.5), 96.3 \%( \pm 4.6)$, and $70.5 \%( \pm 11.5)$, respectively.

Conclusion This proposed method may be a potential tool to diagnose AD pathology with the current clinical criteria.

Psychiatry Investig 2015;12(1):92-102

Key Words Magnetic resonance imaging, Alzheimer's disease, Diagnosis, Support vector machines.

\section{INTRODUCTION}

Alzheimer's disease $(\mathrm{AD})$, which is becoming the most prevalent neurodegenerative disorder with aging of the population, leads to an irreversible decline in memory, cognition and thinking ability. ${ }^{1,2}$ Mild cognitive impairment (MCI) and subjective memory impairment (SMI) have been proposed as intermediate states of $\mathrm{AD}$ from cognitively normal elderly (CNE) people. ${ }^{3,4} \mathrm{MCI}$ particularly has been conceived as a clinically prodromal state with objective cognitive decline. ${ }^{5}$

Received: September 2, 2013 Revised: December 29, 2013

Accepted: December 30, 2013 Available online: January 12, 2015

$\triangle$ Correspondence: Chi-Woong Mun, $\mathrm{PhD}$

Department of Biomedical Engineering/u-HARC, Inje University, Building A, 197 Inje-ro, Gimhae 621-749, Republic of Korea

Tel: +82-55-320-3297, Fax: +82-55-327-3292, E-mail: mcw@inje.ac.kr

(a) This is an Open Access article distributed under the terms of the Creative Commons Attribution Non-Commercial License (http://creativecommons.org/licenses/bync/3.0) which permits unrestricted non-commercial use, distribution, and reproduction in any medium, provided the original work is properly cited.
SMI is accepted as within the normal range on a clinical neuropsychological rating scale. ${ }^{6}$ Considering the decline in memory and cognitive function associated with aging, SMI includes the potential risk of conversion to $\mathrm{MCI}$ and $\mathrm{AD} .^{7}$ The pathological AD markers include extracellular accumulations of aggregated amyloid- $\beta$ peptide ${ }^{8}$ and intracellular $\tau$-associated neurofibrillary tangles (NFTs). ${ }^{9}$ NFTs are predominantly observed in hippocampal formations in autopsy models corresponding with $\mathrm{AD}$ staging, whereas amyloid plaques show irregular distribution patterns. ${ }^{10,11} \mathrm{~T} 1$-weighted brain structural magnetic resonance imaging (MRI) enhances the contrast between gray matter (GM) and white matter (WM). A high correlation between regional NFT counts and volume atrophy in the hippocampus (HP) has been reported using this technique. ${ }^{12}$ Thus, brain MRI is useful to indirectly evaluate structural changes by AD pathology. Another MRI technique, diffusion tensor imaging (DTI), allows for in vivo examination of microscopic random motion of water mole- 
cules, which represent the orientation and directionality of surrounding tissues, and provides quantitative information on microstructural brain integrity ${ }^{13,14}$ Although interpretation for the results of these techniques with respect to the causal mechanisms of $\mathrm{AD}$ pathogenesis may ambiguous, the techniques are suitable as complementary indicators to elucidate neural degeneration in AD. General group-level comparisons using MRI [e.g., voxel-based morphometry (VBM) and tract-based spatial statistics (TBSS)] provide fascinating insights into disease-related alterations. However, these results are difficult to apply to clinical neuroradiology at the individual level, especially in the identification of physiological conditions. ${ }^{15}$ Recently, machine-learning methods that can sort individual results into different classes using neuroimaging data have been introduced. ${ }^{15-22}$ Conceptually, these require the learning of class patterns from reference group data, called a training set, to distinguish the individuals from the so-called test set. Therefore, it is important to determine how well machine-learning can create the feature space to perform the classification and how well the feature is able to represent individual conditions. Support vector machine (SVM) is based on the optimal hyperplane to maximize the separation between classes, and it shows the high classification performance compared with other methods (e.g., linear discriminant analysis and logistic regression). ${ }^{20-22}$ Therefore, the objectives in this study were, first, to investigate the macroscopic cortical and subcortical GM changes by structural volumetry and microscopic brain alterations by DTI analyses across preclinical and AD groups to identify MRI markers. Second, we classified MRI markers using SVM to propose an automated MRI-based diagnostic method.

\section{METHODS}

\section{Subjects}

This study was approved by the Pusan National University Hospital Institutional Review Board. All subjects signed written informed consent forms. Twenty-seven patients with SMI, 18 patients with MCI, and 27 patients with $\mathrm{AD}$ were recruited from the memory impairment clinic of Pusan $\mathrm{Na}$ tional University Hospital from November 2010 to March 2012. The demographic features of the subjects and the results of neuropsychological tests based on Korean version of Consortium to Establish a Registry for Alzheimer's Disease assessment packet (CERAD-K), ${ }^{23}$ Clinical Dementia Rating scale $(\mathrm{CDR})^{24}$ and Clinical Dementia Rating Some of Box (CDR$\mathrm{SOB})^{25}$ are summarized in Table 1 . The inclusion criteria for SMI were 1) sustained subjective memory complaints; 2) normal general cognition [within -1.5 standard deviation (SD) of age- and education-adjusted norms on the Korean version of the Mini Mental State Examination (K-MMSE) ${ }^{26}$ and a score $>26$ ]; 3) intact activities of daily living (ADL); and 4) no abnormalities (within -1.5 SD of age- and education-adjusted norms) detected on a comprehensive neuropsychological battery. MCI was diagnosed according to the revised Petersen's criteria: $\left.{ }^{5} 1\right)$ subjective cognitive complaints, preferably corroborated by an informant; 2) objective impairment on any of the neuropsychological tests (at least 1.5 SD below the mean for age- and education-matched norms); 3) preserved global intellectual function; 4) preserved or minimal impairment in $\mathrm{ADL}$; and 5) no dementia. AD was diagnosed according to the criteria of the National Institute of Neurological and Communicative Disorders and Stroke and the Alzheimer's Disease

Table 1. Demographic information of the subjects

\begin{tabular}{|c|c|c|c|c|}
\hline & SMI (N=27) & $\operatorname{MCI}(\mathrm{N}=18)$ & $\mathrm{AD}(\mathrm{N}=27)$ & p-value \\
\hline Age, years" & $67.33 \pm 6.11$ & $71.77 \pm 7.02$ & $72.07 \pm 6.66^{*}$ & 0.03 \\
\hline Gender radio $(\mathrm{F} / \mathrm{M})^{* *}$ & $18 / 9$ & $15 / 3$ & $19 / 8$ & 0.47 \\
\hline Education, years ${ }^{*}$ & $8.67 \pm 4.36$ & $6.56 \pm 5.29$ & $8.50 \pm 5.58$ & 0.34 \\
\hline $\mathrm{MMSE}^{*}$ & $26.74 \pm 1.97$ & $23.11 \pm 5.09^{\ddagger}$ & $16.67 \pm 5.10^{\dagger \pi}$ & $<0.01$ \\
\hline $15-\mathrm{KBT}^{*}$ & $10.6 \pm 1.3$ & $9.5 \pm 3.3$ & $7.4 \pm 3.2^{+\pi}$ & $<0.01$ \\
\hline Constructional praxis ${ }^{\#}$ & $9.9 \pm 1.6$ & $9.3 \pm 1.9$ & $8.7 \pm 2.1^{*}$ & 0.03 \\
\hline Word list recall ${ }^{*}$ & $5.5 \pm 1.7$ & $3.0 \pm 1.7 \S$ & $0.6 \pm 0.9^{+\pi}$ & $<0.01$ \\
\hline Constructional recall ${ }^{*}$ & $5.7 \pm 3.6$ & $2.9 \pm 3.0^{\S}$ & $1.8 \pm 1.8^{\dagger}$ & $<0.01$ \\
\hline $\mathrm{FAB}^{\#}$ & $14.2 \pm 1.9$ & $12.7 \pm 2.6 \S$ & $10.9 \pm 3.4^{\dagger}$ & $<0.01$ \\
\hline $\mathrm{CDR}^{\#}$ & $0.4 \pm 0.2$ & $0.55 \pm 0.2$ & $1.2 \pm 0.4^{+\pi}$ & $<0.01$ \\
\hline CDR-SOB ${ }^{*}$ & $1.4 \pm 1.0$ & $2.5 \pm 0.9 \S$ & $5.5 \pm 2.7+\pi$ & $<0.01$ \\
\hline
\end{tabular}

Data are presented as mean \pm standard deviation. Statistics ( $\mathrm{p}$-value) are derived from ANOVA and $\chi^{2}$ test. Indicators mean the significances from Boferroni post-hoc test. ${ }^{*} \mathrm{p}<0.05$ and ${ }^{\dagger} \mathrm{p}<0.01$ between $\mathrm{SMI}$ and $\mathrm{AD},{ }^{\ddagger} \mathrm{p}<0.05$ and ${ }^{\S} \mathrm{p}<0.01$ between SMI and MCI, "p $<0.01$ between MCI and $\mathrm{AD}$, \#by ANOVA followed by Bonferroni post hoc test, ${ }^{* *}$ by $\chi^{2}$ test. SMI: Subjective Memory Impairment, MCI: Mild Cognitive Impairment, AD: Alzheimer's disease, MMSE: Mini Mental State Examination, 15-KBT: 15-item Korean Boston Naming Test, FAB: Frontal Assessment Battery, CDR: Clinical Dementia Rating Scale, CDR-SOB: Clinical Dementia Rating Some of Box 
and Related Disorders Association. ${ }^{27}$ We applied the following exclusion criteria to all subjects: 1 ) other neurodegenerative diseases; 2) major depressive disorder according to the Korean version of Mini International Neuropsychiatric Interview (MINI-K) ${ }^{28,29}$ or other psychiatric illness; 3 ) intracranial space-occupying lesion; 4) aphasia or other language barrier; 5) MRI contraindications or known claustrophobia; 6) active substance abuse disorders; 7) severe systemic disease; 8) prominent visual or hearing impairment. We also excluded participants with moderate or severe white matter hyperintensitiy (WMH) on brain MRI to minimize the vascular etiologic impact on SMI. The presence of moderate or severe $\mathrm{WMH}$ were defined as WMH on fluid attenuated inversion recovery images that fulfilled the following criteria: 1) periventricular WMH (caps or rim) $>5 \mathrm{~mm}$ and 2) deep WMH consistent with an extensive WM lesion or diffusely confluent lesion $\geq 10 \mathrm{~mm}$ in maximum diameter.

\section{MRI data acquisition}

Subjects were examined using a multi-sequence MRI protocol on a 3T Siemens Trio Tim system (Erlangen, Germany). High resolution T1-weighted images (T1WI) were acquired using a three-dimensional magnetization-prepared rapid gradient-echo sequence with the following parameters: repetition time $(\mathrm{TR})=1800 \mathrm{~ms}$, echo time $(\mathrm{TE})=2.07 \mathrm{~ms}$, inversion time $(\mathrm{TI})=900 \mathrm{~ms}$, flip angle $=12^{\circ}$, acquisition matrix $=$ $256 \times 256$, field of view $(F O V)=250 \times 250 \mathrm{~mm}^{2}$, slice thickness $=1 \mathrm{~mm}$, and total number of slices $=256$. DTI data were acquired using an echo-planar imaging sequence applied in diffusion-weighted gradients along 30 non-collinear directions ( $\mathrm{b}$ value $=600 \mathrm{~s} / \mathrm{mm}^{2}$ ) and one volume without diffusion weighting $\left(\mathrm{b}_{0}\right)$ using the following acquisition parameters: number of average $=2, \mathrm{TR}=6200 \mathrm{~ms}, \mathrm{TE}=85 \mathrm{~ms}$, flip angle $=90^{\circ}$, acquisition matrix $=128 \times 128, \mathrm{FOV}=230 \times 230 \mathrm{~mm}^{2}$, and slice thickness $=3 \mathrm{~mm}$.

\section{MRI data processing}

\section{Structural image processing}

Whole-brain structural analyses of cerebral cortical thickness and subcortical volume based on T1WI were performed using FreeSurfer v.5.1 (http://surfer.nmr.mgh.harvard.edu/). ${ }^{30,31}$ As a fully automated image-processing tool for macroscopically visible brain structures, FreeSurfer constructs models of brain tissue surfaces and subcortical areas, and is useful to measure cerebral cortical thickness and subcortical volumes. Briefly, the images are processed with a head motion correction, a non-uniform intensity correction for MR acquisition artifacts, affine transformation to the MNI 305 template, further intensity normalization across brain tissues, and strip- ping of non-brain areas. The preprocessed brain image was non-linearly transformed to align with Gaussian classifier atlas model, which was designated with the voxel labels based on neuroanatomical probabilistic information estimated from a large training dataset. Images were segmented into cortical and subcortical structures using iterative methods to compute anatomical probabilities by combining intensity distribution with spatial relationships between subject and probabilistic atlas for each voxel until no any changes are detected in two consecutive iterations. Brain volume per hemisphere was initially covered with triangular tessellation to construct surface models across the different tissue types and to define the surface vertices. The surface defects from previous segmentation steps are topologically refined for accurate boundary connectivity, and then the intensity gradient determined WM surfaces as well as pial surfaces. Local cortical thickness was measured as the average distance between vertices corresponding to the brain anatomy of these two surface models. The cortical thickness and subcortical volume normalized with intracranial volume were compared using multivariate analysis of covariance (MANCOVA) with age entered as a covariate followed by Bonferroni post hoc test for feature selection of the classification.

\section{DTI processing}

The DTI analysis to evaluate microstructural WM integrity was performed with FMRIB Software Library (FSL, http:// www.fmrib.ox.ac.uk/fsl) package. ${ }^{32}$ Raw diffusion-weighted images were linearly aligned to $b_{0}$ image, followed by removing the non-brain tissues using the brain extraction tool (BET). ${ }^{33}$ The diffusion tensor was calculated at each voxel to generate the fractional anisotropy (FA) and mean diffusivity (MD) images. The WM segmentation was based on the subsequent TBSS procedures. ${ }^{34}$ All individual FA images were linearly and nonlinearly aligned to FMRIB58_FA template. ${ }^{32}$ To create the mean FA skeleton that served as the study-specific template, all aligned FA images were averaged and thinned by local perpendicular non-maximum suppression with FA thresholds of 0.2 to exclude GM and cerebrospinal fluid (CSF). The resulting skeleton represented the center of the common WM tracts. The FA values nearest the mean FA skeleton from all aligned images were projected onto this skeleton. The skeleton was labeled to identify the $50 \mathrm{WM}$ regions with reference to the JHU ICBM-DTI-81 WM atlas ${ }^{35}$ and to generate the binary masking images of WM region of interest (ROI). These masking images in standard space were transformed back into each subject's native space using the inverse normalization with skeleton deprojection and then multiplied by the native DTIs to obtain the average DTI values (i.e., FA and MD) within the ROI. Next, the $b_{0}$ image for 
each subject was linearly registered to the skull stripped brain from T1WI, and then the transform matrix was applied to the DTIs. The subcortical GM showing volume atrophy was superimposed over the aligned DTIs in T1WI space to evaluate the microstructural changes, and the average DTI values were calculated within ROI for each hemisphere. The statistical analysis for DTI values was performed with the same design as described above.

\section{Parameter selection and feature analysis}

We included structural parameters (i.e., cortical thickness and subcortical volumes), DTI parameters (i.e., FA, MD values and the DTI ratio between MD and FA) and a multimodal parameter as the ratio between MD value and volume for the classification. To identify the most reliable diagnostic imaging markers between features (i.e., regions and parameters) and to reduce the amount of processing data, the feature analysis was performed with support vector machine-recursive feature elimination (SVM-RFE) and principle component analysis (PCA). SVM-RFE estimates the feature's ranking by comparing the magnitudes of the weighting values $\left(\omega^{2}\right)$ from the linear SVM classifier for each feature and recursively removing the features with the smallest weighting values, which means the least significant for classification at each step. ${ }^{36}$ Based on the assigned priority, PCA was performed to obtain the two standardized scores derived from the two top-ranked eigenvalues that reduced the data dimensions but maintained the information of the original data. The PCA sources were selected in consecutive order from the most- to the least relevant feature until the highest accuracy for classification was detected in the following method.

\section{Classification using SVM}

Feature maps were constructed using nonlinear SVM with the radial basis function (RBF) kernel. ${ }^{37}$ The advantages of this classifier are identification of reliable data points, called support vectors, and transformation into high-dimensional space with a kernel trick to identify nonlinear boundaries when the classes are not linearly separable. The RBF kernel involved the two parameters, denoted $\gamma$ and C, to nonlinearly transform the datasets into high-dimensional space. The parameter $\gamma$ means the kernel width and the parameter $C$ represents the tradeoff between the maximization of discriminative margin and minimization of the misclassification error. A grid search was performed with a search range of $\left(2^{-5}\right.$ and $\left.2^{5}\right)$ using a 10-fold cross-validation to determine the optimal two parameters. Four feature maps were constructed with respect to classification performance levels for all three groups and pairs of groups. Eighty percent of each group was randomly sampled to form a training set for learning the feature maps, and the remaining $20 \%$ was used as the test set for cross-validation. The procedures were repeated 1,000 times, with data resampling and grid search, and the average accuracy was evaluated as the classification performance.

\section{RESULTS}

\section{Differences in cortical thickness and subcortical volume}

Seventeen GM structures showed the significant differences on the neurodegenerative states compared with the other groups. Cortical thinning was found in the medial temporal (i.e., bilateral entorhinal cortex and bilateral fusiform cortex) regions, bilateral temporal pole and left insula cortex in patients with $\mathrm{AD}$ compared with those in patients with SMI. Patients with MCI showed intermediate thickness in these cortices except the right temporal pole region (Figure 1). Volume atrophy occurred in the HP, amygdala (AMG) and nucleus accumbens (NA) in both hemispheres in AD patients compared with SMI. Atrophy between patients with MCI and $\mathrm{AD}$ was also observed in the left HP and AMG. Volumes of the right $\mathrm{HP}$ and AMG were significantly decreased in MCI patients compared to those with SMI. In contrast, ventricular volumes (i.e., lateral ventricles, third ventricles, and bilateral inferior lateral ventricles) were significantly enlarged in patients with $\mathrm{AD}$ compared with those of patients with SMI and MCI (Figure 2).

\section{Microstructural damage in WM tracts and subcortical GM}

Neurodegenerative WM damages were associated with a

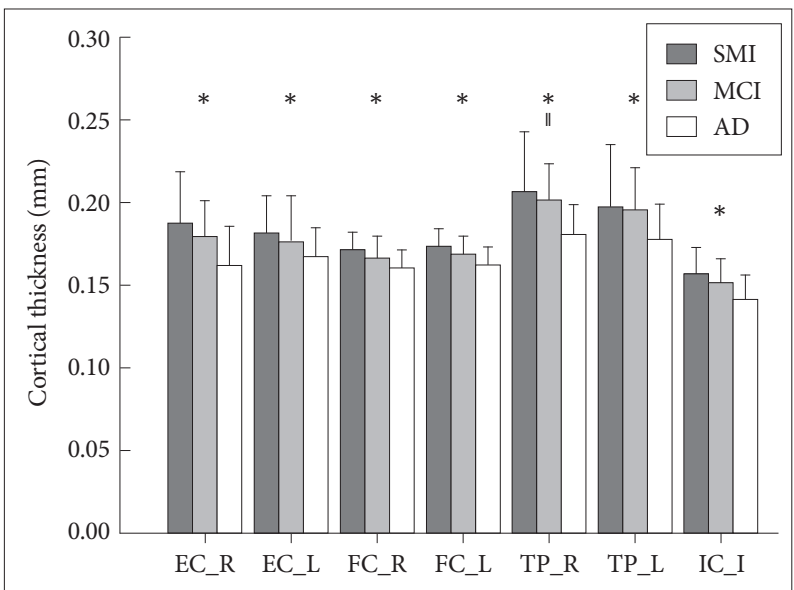

Figure 1. Significantly different cortical thickness $(\mathrm{mm})$ in patients with SMI, $\mathrm{MCl}$ and $\mathrm{AD}$. Indicators mean the significances from MANCOVA followed by Boferroni post-hoc test. ${ }^{*} p<0.05$ between SMI and $A D$, "p<0.05 between $\mathrm{MCl}$ and $A D$. SMI: subjective memory impairment, $\mathrm{MCl}$ : mild cognitive impairment, $\mathrm{AD}$ : Alzheimer's disease, EC: entorhinal cortex, FC: fusiform cortex, TP: temporal pole, IC: insular cortex, R: right, L: left. 
FA decrease and an MD increase. A significant $(\mathrm{p}<0.05)$ or progressive decrease in FA values was observed in $12 \mathrm{WM}$ ROIs across the three groups (Figure 3 ). These regions included the bilateral anterior corona radiate (ACR), two cingulums of cingulate gyrus and hippocampal portions, the fornix of cres (Fx), the corpus callosum (CC) of genu, body, and splenium portions and the left uncinate fasciculus (UF). The FA values in patients with $\mathrm{AD}$ were significantly decreased in all of these regions compared with those in patients with SMI. Compared to patients with MCI, the FA values in bilateral cingulum of the cingulate gyrus (CGC) were also significantly decreased in $\mathrm{AD}$. The remaining $10 \mathrm{WM}$ regions, except the bilateral ACR, showed significant differences in $\mathrm{MD}$ values between $\mathrm{AD}$ and $\mathrm{SMI}$ patients (Figure 4). In particular, MD values of $\mathrm{AD}$ patients were higher in the body of corpus cal- losum (BCC), the two cingulums and Fx than in MCI patients. MD values of MCI patients were increased only in the left cingulum of hippocampus (CGH) compared with SMI patients. Along with these results, the microscopic damage in the subcortical GM showing volume atrophy were reflected by an increase in MD values, but not in FA values (Figure 5). Patients with $\mathrm{AD}$ showed the MD increase in all regions, whereas patients with MCI showed an increase in the left HP alone compared to patients with SMI. All regions except the $\mathrm{NA}$ and right $\mathrm{AMG}$ showed higher $\mathrm{MD}$ values in patients with $\mathrm{AD}$ than in those with MCI.

\section{Parameter selection and feature extraction}

From the multivariate statistics of the imaging analysis, SVM-RFE assigned the ranking of features as more or less re-

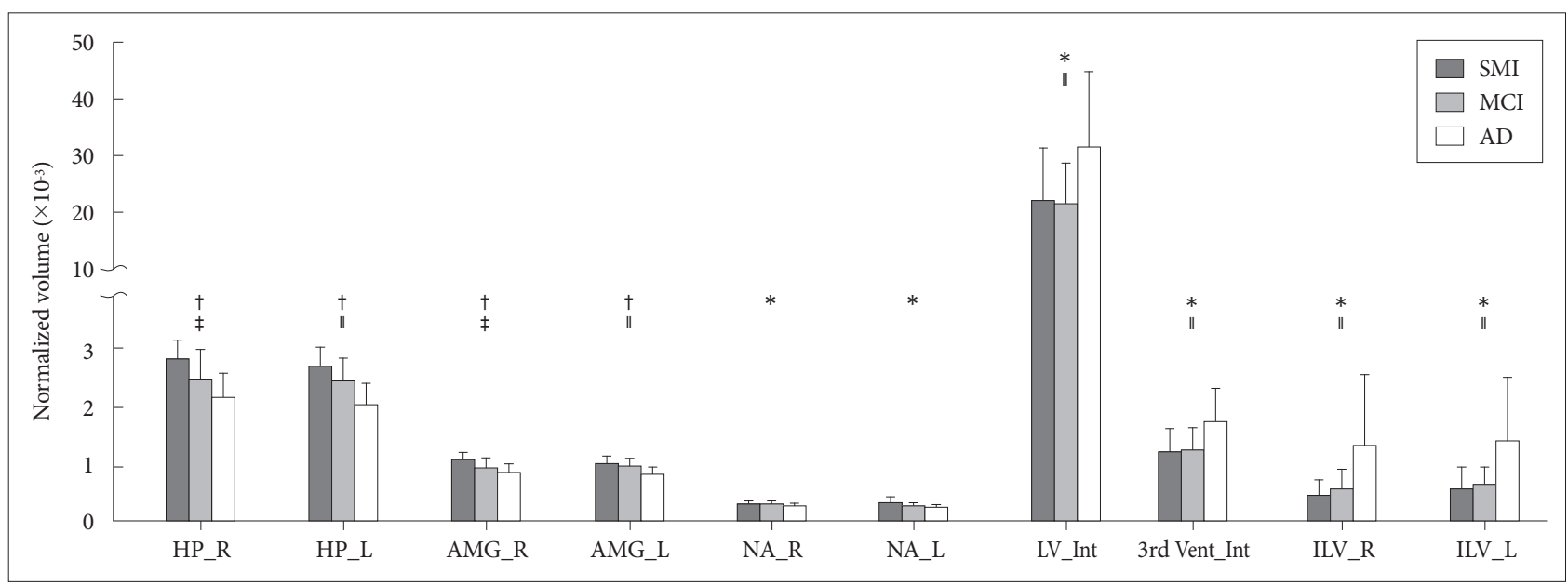

Figure 2. Significantly different structural volume $\left(\times 10^{-3}\right)$ normalized by intracranial volume in patients with $\mathrm{SMI}$, $\mathrm{MCl}$ and $\mathrm{AD}$. Indicators mean the significances from MANCOVA followed by Boferroni post-hoc test. ${ }^{*} p<0.05$ and ${ }^{\dagger} p<0.01$ between SMI and AD, ${ }^{\ddagger} p<0.05$ between SMI and $\mathrm{MCl}$, "p<0.05 between $\mathrm{MCl}$ and $\mathrm{AD}$. SMI: subjective memory impairment, $\mathrm{MCl}$ : mild cognitive impairment, AD: Alzheimer's disease, HP: hippocampus, AMG: amygdale, NA: nucleus accumbens, LV: lateral ventricle, ILV: inferior lateral ventricle, R: right, L: left, Int: inter hemisphere.

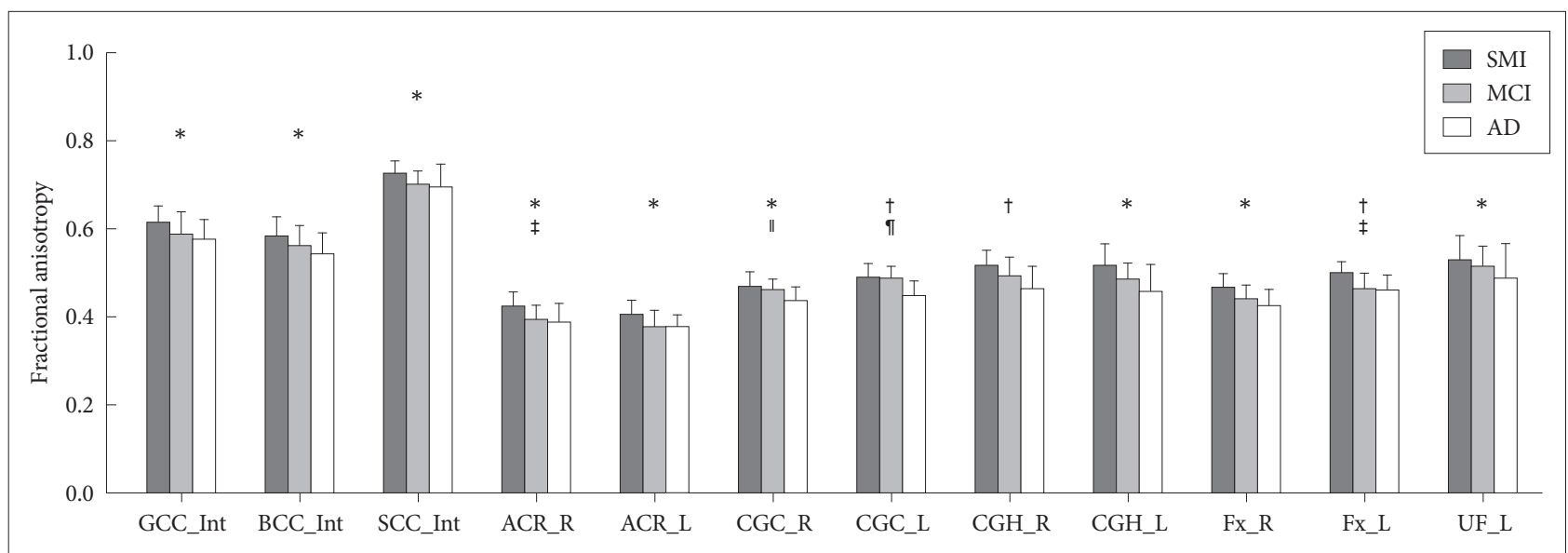

Figure 3. Significantly different FA value of WM ROI in patients with SMI, MCI and AD. Indicators mean the significances from MANCOVA followed by Boferroni post-hoc test. ${ }^{*} p<0.05$ and ${ }^{\dagger} p<0.01$ between SMI and AD, ${ }^{\ddagger} p<0.05$ between SMI and MCl, "p<0.05 and " $p<0.01$ between $\mathrm{MCl}$ and $\mathrm{AD}$. SMI: subjective memory impairment, $\mathrm{MCl}$ : mild cognitive impairment, AD: Alzheimer's disease, GCC: genu of the corpus callosum, BCC: body of the corpus callosum, SCC: splenium of the corpus callosum, ACR: anterior corona radiate, CGC: cingulum of the cingulate gyrus, CGH: cingulum of the hippocampus, Fx: fornix of cres, UF: uncinate fasciculus, R: right, L: left, Int: inter-hemisphere. 


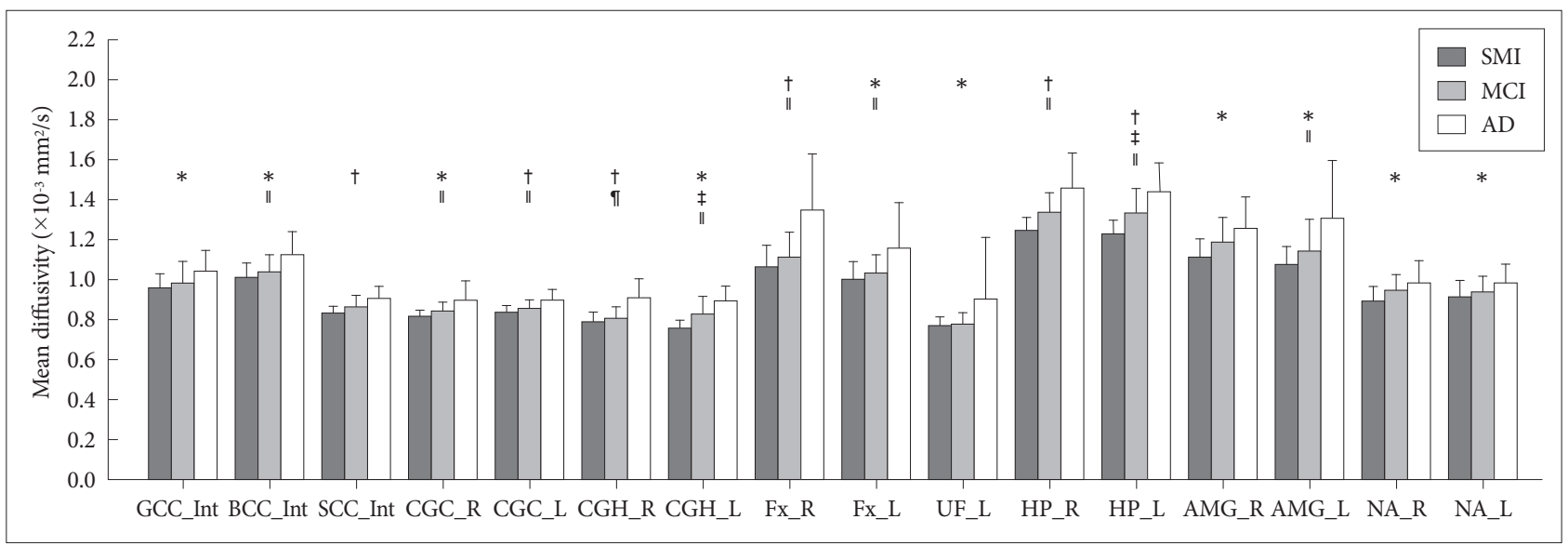

Figure 4. Significantly different $\mathrm{MD}$ values $\left(\times 10^{-3} \mathrm{~mm}^{2} / \mathrm{s}\right)$ of $\mathrm{WM} \mathrm{ROI}$ in patients with $\mathrm{SMI}, \mathrm{MCl}$ and $\mathrm{AD}$. Indicators mean the significances from MANCOVA followed by Boferroni post-hoc test. ${ }^{*} p<0.05$ and ${ }^{\dagger} p<0.01$ between SMI and $A D,{ }^{\ddagger} p<0.05$ between SMI and MCI, " $p<0.05$ and Ip $<0.01$ between $\mathrm{MCl}$ and $\mathrm{AD}$. SMl: subjective memory impairment, $\mathrm{MCl}$ : mild cognitive impairment, AD: Alzheimer's disease, GCC: genu of the corpus callosum, BCC: body of the corpus callosum, SCC: splenium of the corpus callosum, ACR: anterior corona radiate, CGC: cingulum of the cingulate gyrus, CGH: cingulum of the hippocampus, Fx: fornix of cres, UF: uncinate fasciculus, HP: hippocampus, AMG: amygdala, NA: nucleus accumbens, R: right, L: left, Int: inter-hemisphere.

liable diagnostic imaging markers for classification. The $\mathrm{MD}$ value in the left HP was identified as the most relevant feature and the volume of the right NA was the least relevant feature for the classification across all groups. MD values in the left $\mathrm{CGH}$ (SMI vs. $\mathrm{AD}$ ) and $\mathrm{MD}$ values in the right CGH (MCI vs. $\mathrm{AD}$ ) were labeled as the most relevant features, respectively. Finally, the top-ranked reliable feature between SMI and MCI was the MD value of the right HP. The most accurate cross validation was ensured by applying the features in consecutive order to discriminate the classes. First, feature maps were constructed for the entire group classification and were implemented using two PCA standardized scores based on the top-ranked features, including MD values in the left HP and the right CGH, the multimodal ratio in the left HP, DTI ratio in the left CGH, the FA value in the left ACR and the DTI ratio in the left CGC. Second, features that showed the highest accuracy for cross validation between SMI and $\mathrm{AD}$ were identified using the five features, including MD values in the left CGH, left HP, and left AMG; the multimodal ratio in the left HP; and the DTI ratio in the left CGH. Third, the most accurate differentiation between $\mathrm{MCI}$ and $\mathrm{AD}$ was achieved using five features including the MD value in the right CGH, the FA value in the left ACR, the DTI ratio in the left CGC, FA values in the left Fx and the right ACR. Finally, the feature map for distinguishing SMI and MCI was created using only two features, MD values in the right $\mathrm{HP}$ and the DTI ratio in the splenium of CC (SCC).

\section{SVM classification}

A random sample of $80 \%$ of each group was used to construct a feature map and the remaining $20 \%$ was included for cross-validation between the clinical assessment and imaging analysis. Because this procedure was repeated 1000 times to achieve global accuracy, the feature maps were differently configured at each step, depending on the resampled training set (Figure 5). The results of classification yielded an average accuracy of $70.5 \%( \pm 11.5)$ for the three groups. Moreover, SMI and MCI could be distinguished from each other with $84.4 \%( \pm 13.8)$ accuracy, followed by $86.9 \%( \pm 10.5)$ accuracy in distinguishing MCI from AD. The highest accuracy, $96.3 \%$ $( \pm 4.6)$, was achieved for distinguishing SMI from AD.

\section{DISCUSSION}

This study was designed to investigate the macro- and microscopic changes in patients with AD pathology using MRI and to distinguish the disease severity of $\mathrm{AD}$ from $\mathrm{SMI}$ and MCI using a SVM classification based on imaging markers.

\section{Structural measures}

The patterns of cortical thinning and volume atrophy corresponded to the disease progression. Cortical thinning occurred in the medial temporal regions, the bilateral temporal pole, and the left insula cortex in $\mathrm{AD}$ patients compared to SMI patients. MCI patients also showed some cortical thinning compared to SMI patients, but not significant. These patterns were consistent with previous studies that included $\mathrm{CNE}$ and patients with $\mathrm{MCI}$ and $\mathrm{AD}{ }^{38,39}$ The medial temporal regions play a vital role in the functions of declarative or long-term memory. Cortical thinning in these regions is congruent with the clinical symptom of memory deficits in $\mathrm{AD}$ pathology. In particular, the medial temporal regions and temporal pole cortices are initially affected by $\tau$-associated NFTs. ${ }^{10,40}$ The insular cortex is associated with diverse func- 


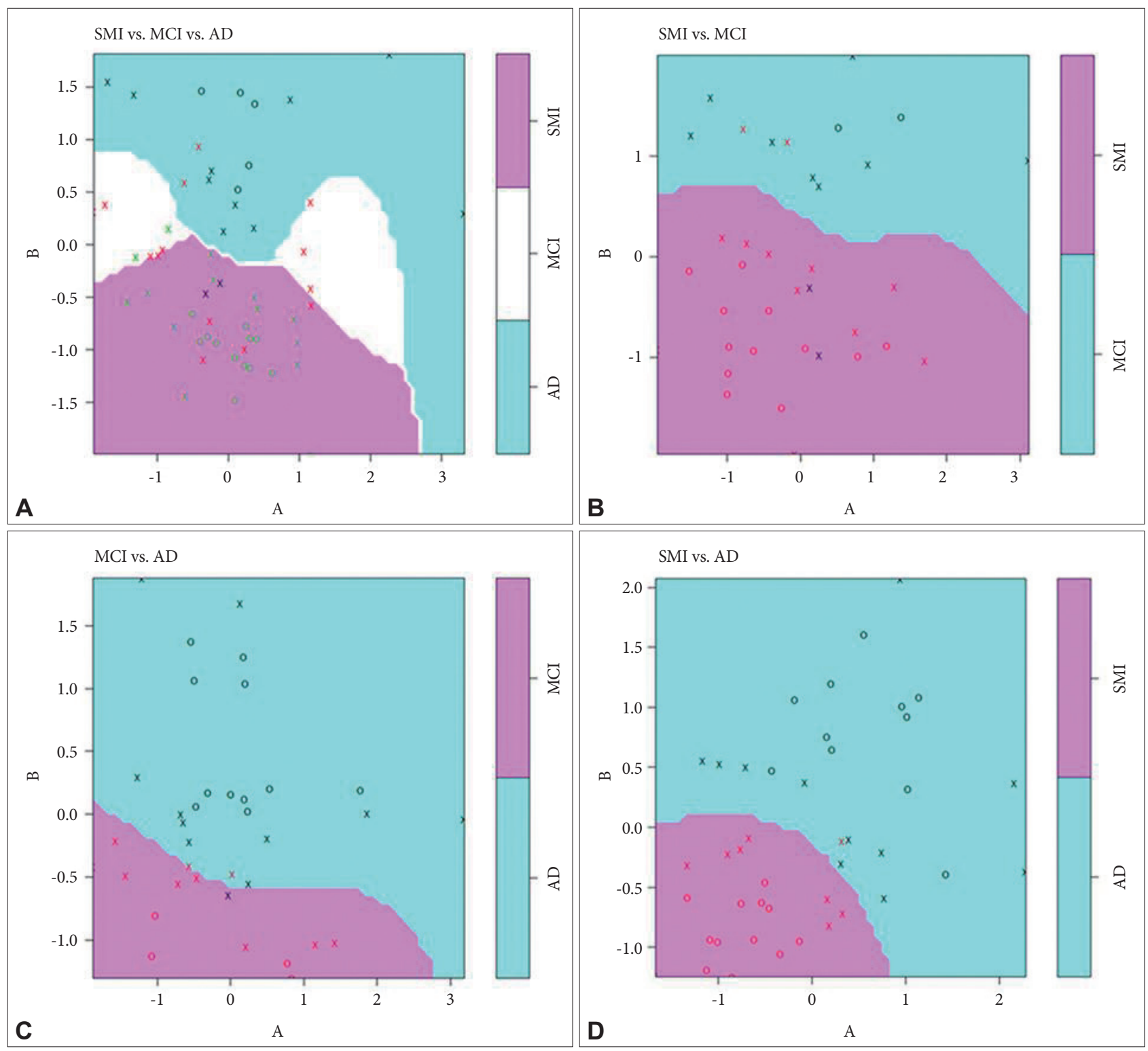

Figure 5. Examples of two-dimensional feature map using the ninth data set derived from factor extraction. A: Black indicators (i.e., ' $\circ$ ' and ' $X$ ') exhibit the Alzheimer's disease (AD) dataset, green represents subjective memory impairment (SMI), and red is mild cognitive impairment $(\mathrm{MCl})$. B: Black indicators are the $\mathrm{MCl}$ dataset, and red is SMI. C: Black indicators are the AD dataset, and red is $\mathrm{MCl}$. D: Black indicators are the AD dataset, and red is SMI. The symbol ' $x$ ' in the dataset is a support vector to determine the hyperplane between the groups. Colored background visualizes partitions of the classified group regions. The horizontal axis represents the first standard score derived from the PCA feature extraction, and the vertical axis indicates the second score.

tions ranging from motor control and pain perception to emotional processing. ${ }^{39}$ In particular, the anterior insular cortex is regarded as the limbic-related cortex that controls emotion and memory functions. ${ }^{41,42}$ Considering that higher NFT density has been found in the agranular cortex of the anterior insular portion than in other portions in patients with $\mathrm{AD}$ progression, ${ }^{43}$ cortical thinning of the insular regions is thought to originate from secondary damage by $\tau$ pathology after NFTs have accumulated in the medial temporal regions and temporal pole cortices. We also found a progressive decrease in subcortical volumes (i.e., HP, AMG, and NA) and observed a volumetric increase in the ventricular system along with disease severity. Many studies have found large deposits of NTFs and significant HP atrophy in AD patients. ${ }^{12,44}$ The functions of HP are mainly divided into forming memories and spatial memory. In particular, the left HP is proposed to support memorizing experienced events, whereas the right $\mathrm{HP}$ is involved in location-associated memory. ${ }^{45}$ Thus, our results suggest that $\mathrm{AD}$ patients might find it more difficult to recall the personal experiences than do the others and that MCI patients would complain of deficits in recording spatial memory significantly more than SMI. AMG vol- 
ume also decreased, similar to the HP atrophy. AMG has been reported to show pathological changes similar to those observed in the HP, and a high correlation has been found between performance in global cognitive functions (i.e., MMSE) and AMG volume. ${ }^{46,47}$ Therefore, AMG atrophy is also suggested as an earlier biomarker of $\mathrm{AD}$ pathology. The other atrophied regions were the bilateral NA. These structures receive afferents from (or project to) the limbic system through the mesolimbic pathway as well as the fornix-fimbria fibers and contribute to integrating information related to cognitive function. ${ }^{48,49}$ Given these previous and present results of significant volumetric damages in $\mathrm{AD}$ patients, we speculate that NA atrophy may have originated from secondary degeneration of HP. The ventricular system adjacent to the medial temporal lobe was significantly enlarged, with a corresponding volume loss in patients with $\mathrm{AD}$ compared with the other groups. A previous study reported that the hemispheric atrophy and ventricular enlargement were greater in those who clinically converted to a more severe form of cognitive impairment (e.g., from $\mathrm{CNE}$ to $\mathrm{MCI}$ or $\mathrm{AD}$ and from $\mathrm{MCI}$ to $\mathrm{AD}$ ) or progressed faster to a more severe $\mathrm{AD}$ stage (e.g., from mild- to moderate $\mathrm{AD}$ ) than those whose clinical conditions was unchanged. ${ }^{50}$ Additionally, the ventricular enlargement was highly related to both cortical NTFs and amyloid plaques, to the rate of HP atrophy and hippocampal NTFs in patients with $\mathrm{AD}$ until death. ${ }^{51}$ These results suggest that ventricular volume changes are effective to assess the treatment response and to understand the disease progression. Taken together, these findings suggest that the ventricular enlargement may be mirrored by the cortical abnormalities and be a sensitive indicator of subsequent clinical severity due to prior damage in the medial temporal lobe.

\section{DTI parameters}

WM damage was detected in progressive FA decrease, together with MD increase in the corpus callosum, the two cingulums, Fx and the left UF across all groups. The volume atrophy observed in the subcortical ROI was mirrored by a concomitant $\mathrm{MD}$ increase. A lower FA value together with higher MD value in WM tracts possibly resulted from alterations in tissue cytoarchitecture (i.e., axon density and myelin integrity), ${ }^{52}$ which also reflects disruption of structural barriers to water diffusivity or enlargement of the extracellular space. ${ }^{53} \mathrm{FA}$ is low in GM due to the complex orientation of cell membranes and multiple crossing fibers (i.e., local dendrites and axons) compared with FA in WM, where water motion is restricted in the dominant direction by the aligned axons. The subtle FA decrease underlying microscopic GM integrity may be difficult to interpret. In contrast, $\mathrm{MD}$ value indicates the magnitude of all directional diffusivity and any change is influenced by the relative spatial extent, not by structural direction. ${ }^{54}$ That is, the MD increase in GM may represent a loss of neuropil including dendrites and unmyelinated axons surrounding neuronal cell bodies, therefore, it may be more useful to identify the microstructural GM damage. Previous DTI studies have reported differences in FA and $\mathrm{MD}$ values between patients with $\mathrm{AD}$ and CNE based on with two main pathophysiological hypotheses. ${ }^{55-60}$ Some researchers have suggested that alterations in WM with disease severity may involve a posterior-to-anterior gradient parallel to the pattern of GM atrophy from the limbic to the frontal and isocortical areas based on $\tau$-pathology; that is, WM disruptions result from anterograde or Wallerian degeneration (WD) secondary to distal GM pathology ${ }^{56-58}$ In contrast, WM degeneration may follow an anterior-to-posterior gradient; that is, it progresses from late-myelinating tracts to early-myelinating tracts by retrogenesis. ${ }^{59,60}$ In this study, WM disruption in the anterior CC and left UF in patients with $\mathrm{AD}$ may be evidence of retrogenesis in $\mathrm{AD}$ pathology. $\mathrm{CC}$ is associated with connectivity of learned, perceptual, and mnemonic cognitive functions between the two hemispheres. ${ }^{61}$ Therefore, $\mathrm{CC}$ damage in patients with $\mathrm{AD}$ may reflect impaired of interhemispheric organization in the entire brain. Additionally, the high $\mathrm{MD}$ value of the $\mathrm{BCC}$, the relatively anterior $\mathrm{CC}$, in patients with $\mathrm{AD}$ compared those with $\mathrm{MCI}$ indicates that latemyelinating WM integrity may be more vulnerable than early-myeliniting tracts of the SCC. These results agree with previous results showing myelin breakdown in the anterior $\mathrm{CC}$, supporting the retrogenesis of $\mathrm{AD}$ pathology, whereas posterior damage of these tracts may occur through WD. As another late-myelinating tract, UF, which connects the inferior frontal lobe with the anterior temporal lobe and limbic system, is also associated with the retrogenesis in patients with $\mathrm{AD} .{ }^{59} \mathrm{DTI}$ fiber-tracking study has found that the ventral-anterior insular cortex involves connectivity with temporal pole via UF and that this structure is also associated with the limbic pathway for regulating emotions. ${ }^{62}$ In keeping with this report, thinning of the left insular cortex was coincident with WM alterations in the left UF in AD patients in our study. Therefore, the hippocampal abnormalities may be linked to damage to WM connectivity and further insular atrophy similar to that in the NA. Taken together, these findings suggest that the DTI results may be associated with both the WD and the retrogenesis.

\section{SVM classification analysis}

Another objective of this study was to distinguish patients with degenerative etiology using the SVM method based on the most relevant MR imaging markers. The present results are comparable to or better than previous classification stud- 
ies in $\mathrm{AD}$ that focused on discriminating between patients with $\mathrm{AD}$ or $\mathrm{MCI}$ and $\mathrm{CNE}$. In studies using structural volumetry only, Magnin et al. achieved $94.5 \%$ mean correct classification using the nonlinear SVM method in 16 patients with $\mathrm{AD}$ (MMSE=23.1) and $22 \mathrm{CNE}(\mathrm{MMSE}=28.5) .{ }^{16}$ Another study demonstrated a maximum $96.4 \%$ correct classification rate using the linear SVM method, when data of 20 patients with confirmed $\mathrm{AD}$ (MMSE=16.7) and $20 \mathrm{CNE}$ (MMSE= 29.0) were assigned as a training set and data from a different center including 14 patients with AD (MMSE=16.1) and 14 controls (MMSE=29.2) were applied as a test set. However, the accuracy decreased to $81.1 \%$ in discrimination between 33 patients with mild $\mathrm{AD}(\mathrm{MMSE}=23.5)$ and $57 \mathrm{CNE}$ (MMSE= 29.1). ${ }^{18}$ Furthermore, previous SVM studies using DTI classified MCI vs. CNE with $91.4 \%$ accuracy $^{19}$ and $93 \%$ accuracy. ${ }^{17}$ The importance of our study can be summarized in three points. 1) The subjects in this study were included to examine brain changes in a narrow cognitive range from SMI to AD. 2) Difference in both macro- and microstructure were analyzed to identify whole-brain damage caused by $\mathrm{AD}$ pathology. 3) Hybrid feature selection methods (ROI based MANCOVA and SVM-RFE) were conducted to determine the relevant regions and imaging parameters for the classification. In this study, the features that best discriminated in correctly classifying each subject were the DTI-MD values. These results suggest that the microstructural GM and WM changes may be closely related to cognitive profile and may also be comparable to features identified in studies based on neurodegenerative etiology. Although the HP is the best-known marker of brain alterations in $\mathrm{AD},{ }^{12,43,63}$ our results indicate that a classification using the selective combination of top-ranked features yielded higher accuracies than any feature alone. This finding suggests that covariance between relevant classification features may accentuate differences among disease conditions. However, contrary to our expectations, which the multimodal ratio may be more reliable for classification than volumes and DTI measures, MD values in these regions were more sensitive features for discrimination. Features that are useful for categorizing subjects into these groups may vary depending on the sample size ${ }^{64}$ therefore, caution is needed when generalizing the results of this study as a feature analysis for $\mathrm{AD}$ specific imaging markers.

\section{Limitations}

There are several limitations in this study. First, the design may have been affected by the relatively small sample size. Although our results show that it is possible to detect brain alterations with macro- and microstructural findings across three groups and to individually classify patients with $\mathrm{AD}$ pathology using a machine-learning model, further validation on a larger sample is required to understand the present results and longitudinal studies should be conducted to clarify the progression of brain changes over time. Another limitation is that CNE were absent from this study. The feature analysis and classification across groups were performed within a relatively narrow spectrum of cognitive disorders. It will necessary to include CNE in a future study to understand which features are reliable and to identify the degenerative pattern of $\mathrm{AD}$ pathology.

\section{Conclusions}

We introduced multimodal MRI measures to identify brain regions and imaging parameters damaged by $\mathrm{AD}$ pathology. Automatic machine learning was conducted to classify the subjects based on imaging markers. A highly accurate classification between the least severe group (SMI) and the most severe group $(\mathrm{AD})$ was achieved at the individual level. Thus, the proposed method may be helpful as an ancillary tool for diagnosing $\mathrm{AD}$ with the current clinical criteria.

\section{Acknowledgments}

This study was supported by a grant of the Korea Healthcare technology R\&D Project, Ministry of Health and Welfare, Republic of Korea (A102065) and of Pusan National University Hospital.

\section{REFERENCES}

1. Cummings JL, Cole G. Alzheimer disease. JAMA 2002;287:2335-2338.

2. Selkoe DJ. Alzheimer's disease is a synaptic failure. Science 2002;298: 789-791.

3. Petersen RC, Doody R, Kurz A, Mohs RC, Morris JC, Rabins PV, et al. Current concepts in mild cognitive impairment. Arch Neurol 2001;58: 1985-1992.

4. van Oijen M, de Jong FJ, Hofman A, Koudstaal PJ, Breteler M. Subjective memory complaints, education, and risk of Alzheimer's disease. Alzheimers Dement 2007;3:92-97.

5. Winblad B, Palmer K, Kivipelto M, Jelic V, Fratiglioni L, Wahlund LO, et al. Mild cognitive impairment-beyond controversies, towards a consensus: report of the International Working Group on Mild Cognitive Impairment. J Intern Med 2004;256:240-246.

6. Petersen RC. Mild cognitive impairment as a diagnostic entity. J Intern Med 2004;256:183-194.

7. Striepens N, Scheef L, Wind A, Popp J, Spottke A, Cooper-Mahkorn D, et al. Volume loss of the medial temporal lobe structures in subjective memory impairment. Dement Geriatr Cogn Disord 2010;29:75-81.

8. Kang J, Lemaire HG, Unterbeck A, Salbaum JM, Masters CL, Grzeschik $\mathrm{KH}$, et al. The precursor of Alzheimer's disease amyloid A4 protein resembles a cell-surface-receptor. Nature 1987;325:733-736.

9. Kitaguchi N, Takahashi Y, Tokushima Y, Shiojiri S, Ito H. Novel precursor of Alzheimer's disease amyloid protein shows protease inhibitory activity. Nature 1988;331:530-532.

10. Braak H, Braak E. Neuropathological stageing of Alzheimer-related changes. Acta Neuropathol 1991;82:239-259.

11. Braak E, Griffing K, Arai K, Bohl J, Bratzke H, Braak H. Neuropathology of Alzheimer's disease: what is new since A. Alzheimer? Eur Arch Psychiatry Clin Neurosci 1999;249(Suppl 3):14-22.

12. Jack CR Jr, Dickson DW, Parisi JE, Xu YC, Cha RH, O’Brien PC, et al. Antemortem MRI findings correlate with hippocampal neuropathology in typical aging and dementia. Neurology 2002;58:750-757. 
13. Pierpaoli C, Basser PJ. Toward a quantitative assessment of diffusion anisotropy. Magn Reson Med 2005;36:893-906.

14. Beaulieu C. The basis of anisotropic water diffusion in the nervous system-a technical review. NMR Biomed 2002;15:435-455.

15. Haller S, Missonnier P, Herrmann FR, Rodriguez C, Deiber MP, Nguyen D, et al. Individual classification of mild cognitive impairment subtypes by support vector machine analysis of white matter DTI. AJNR Am J Neuroradiol 2013;34:283-291.

16. Magnin B, Mesrob L, Kinkingnéhun S, Pélégrini-Issac M, Colliot O, Sarazin M, et al. Support vector machine-based classification of Alzheimer's disease from whole-brain anatomical MRI. Neuroradiology 2009;51:73-83.

17. O’Dwyer L, Lamberton F, Bokde AL, Ewers M, Faluyi YO, Tanner C, et al. Using support vector machines with multiple indices of diffusion for automated classification of mild cognitive impairment. PloS One 2012; 7:e32441.

18. Klöppel S, Stonnington CM, Chu C, Draganski B, Scahill RI, Rohrer JD, et al. Automatic classification of MR scans in Alzheimer's disease. Brain 2008;131:681-689.

19. Haller S, Nguyen D, Rodriguez C, Emch J, Gold G, Bartsch A, et al. Individual prediction of cognitive decline in mild cognitive impairment using support vector machine-based analysis of diffusion tensor imaging data. J Alzheimers Dis 2010;22:315-327.

20. Lehmann C, Koenig T, Jelic V, Prichep L, John RE, Wahlund LO, et al. Application and comparison of classification algorithms for recognition of Alzheimer's disease in electrical brain activity (EEG). J Neurosci Methods 2007;161:342-350

21. Mourão-Miranda J, Bokde AL, Born C, Hampel H, Stetter M. Classifying brain states and determining the discriminating activation patterns: Support Vector Machine on functional MRI data. Neuroimage 2005; 28:980-995.

22. Aguilar C, Westman E, Muehlboeck JS, Mecocci P, Vellas B, Tsolaki M, et al. Different multivariate techniques for automated classification of MRI data in Alzheimer's disease and mild cognitive impairment. Psychiatry Res 2013;212:89-98.

23. Lee JH, Lee KU, Lee DY, Kim KW, Jhoo JH, Kim JH, et al. Development of the Korean version of the Consortium to Establish a Registry for Alzheimer's Disease Assessment Packet (CERAD-K): clinical and neuropsychological assessment batteries. J Gerontol B Psychol Sci Soc Sci 2002;57:P47-P53.

24. Morris JC. The Clinical Dementia Rating (CDR): Current vision and scoring rules Neurology. 1993;43:2412-2414.

25. Hughes CP, Berg L, Danziger WL, Coben LA, Martin RL. A new clinical scale for the staging of dementia. Br J Psychiatry 1982;140:566-572.

26. Kang Y, Na DL, Hahn S. A validity study on the Korean Mini-Mental State Examination (K-MMSE) in dementia patients. J Korean Neurol Assoc 1997;15:300-308.

27. McKhann G, Drachman D, Folstein M, Katzman R, Price D, Stadlan EM. Clinical diagnosis of Alzheimer's disease Report of the NINCDSADRDA Work Group under the auspices of Department of Health and Human Services Task Force on Alzheimer's Disease. Neurology 1984; 34:939-944.

28. Sheehan DV, Lecrubier Y, Sheehan KH, Amorim P, Janavs J, Weiller E, et al. The Mini-International Neuropsychiatric Interview (M.I.N.I.): the development and validation of a structured diagnostic psychiatric interview for DSM-IV and ICD-10. J Clin Psychiatry 1998;59(Suppl 20): 22-33.

29. Choi YH, Yoo SW, Kim YS. Validity of Korean version of the Mini-International Neuropsychiatric Interview. Anxiety Mood 2006;2:50-55.

30. Fischl B, Salat DH, Busa E, Albert M, Dieterich M, Haselgrove C, et al. Whole brain segmentation: automated labeling of neuroanatomical structures in the human brain. Neuron 2002;33:341-355.

31. Fischl B, Salat DH, van der Kouwe AJ, Makris N, Ségonne F, Quinn BT, et al. Sequence-independent segmentation of magnetic resonance images. Neuroimage 2004;23(Suppl 1):S69-S84.
32. Smith SM, Jenkinson M, Woolrich MW, Beckmann CF, Behrens T, Johansen-Berg $\mathrm{H}$, et al. Advances in functional and structural MR image analysis and implementation as FSL. Neuroimage 2004;23(Suppl 1): S208-S219.

33. Smith SM. Fast robust automated brain extraction. Hum Brain Mapp 2002;17:143-155.

34. Smith SM, Johansen-Berg H, Jenkinson M, Rueckert D, Nichols TE, Miller KL, et al. Acquisition and voxelwise analysis of multi-subject diffusion data with tract-based spatial statistics. Nat Protoc 2007;2:499503.

35. Mori S, Wakana S, Van Zijl PCM, Nagae-Poetscher L. MRI atlas of human white matter. Amsterdam: Elsevier Science; 2005.

36. Guyon I, Weston J, Barnhill S, Vapnik V. Gene selection for cancer classification using support vector machines. Mach Learn 2002;46:389-422.

37. Karatzoglou A, Meyer D, Hornik K. Support vector machines in R. J Stat Softw 2006;15:1-28.

38. Singh V, Chertkow H, Lerch JP, Evans AC, Dorr AE, Kabani NJ. Spatial patterns of cortical thinning in mild cognitive impairment and Alzheimer's disease. Brain 2006;129:2885-2893.

39. Yang J, Pan P, Song W, Huang R, Li J, Chen K, et al. Voxelwise metaanalysis of gray matter anomalies in Alzheimer's disease and mild cognitive impairment using anatomic likelihood estimation. J Neurol Sci 2012;316:21-29.

40. Arnold SE, Hyman BT, Flory J, Damasio AR, Van Hoesen GW. The topographical and neuroanatomical distribution of neurofibrillary tangles and neuritic plaques in the cerebral cortex of patients with Alzheimer's disease. Cereb Cortex 1991;1:103-116.

41. Craig AD. How do you feel--now? The anterior insula and human awareness. Nat Rev Neurosci 2009;10:59-70.

42. Mutschler I, Wieckhorst B, Kowalevski S, Derix J, Wentlandt J, SchulzeBonhage A, et al. Functional organization of the human anterior insular cortex. Neurosci Lett 2009;457:66-70.

43. Bonthius DJ, Solodkin A, Van Hoesen GW. Pathology of the insular cortex in Alzheimer disease depends on cortical architecture. J Neuropathol Exp Neurol 2005;64:910-922.

44. Dawe RJ, Bennett DA, Schneider JA, Arfanakis K. Neuropathologic correlates of hippocampal atrophy in the elderly: a clinical, pathologic, postmortem MRI study. PloS One 2011;6:e26286.

45. Burgess N, Maguire EA, O'Keefe J. The human hippocampus and spatial and episodic memory. Neuron 2002;35:625-641.

46. Cuenod CA, Denys A, Michot JL, Jehenson P, Forette F, Kaplan D, et al. Amygdala atrophy in Alzheimer's disease: an in vivo magnetic resonance imaging study. Arch Neurol 1993;50:941-945.

47. Basso M, Yang J, Warren L, MacAvoy MG, Varma P, Bronen RA, et al. Volumetry of amygdala and hippocampus and memory performance in Alzheimer's disease. Psychiatry Res 2006;146:251-261.

48. Cauda F, Cavanna AE, D’agata F, Sacco K, Duca S, Geminiani GC. Functional connectivity and coactivation of the nucleus accumbens: a combined functional connectivity and structure-based meta-analysis. J Cogn Neurosci 2011;23:2864-2877.

49. O'Donnell P. Ensemble coding in the nucleus accumbens. Psychobiology 1999;27:187-197.

50. Jack CR Jr, Shiung MM, Gunter JL, O'brien PC, Weigand SD, Knopman DS, et al. Comparison of different MRI brain atrophy rate measures with clinical disease progression in AD. Neurology 2004;62:591600.

51. Silbert LC, Quinn JF, Moore MM, Corbridge E, Ball MJ, Murdoch G, et al. Changes in premorbid brain volume predict Alzheimer's disease pathology. Neurology 2003;61:487-492.

52. Sotak $\mathrm{CH}$. The role of diffusion tensor imaging in the evaluation of ischemic brain injury-a review. NMR Biomed 2002;15:561-569.

53. Ramani A, Jensen JH, Helpern JA. Quantitative MR Imaging in Alzheimer Disease. Radiology 2006;241:26-44.

54. Pierpaoli C, Jezzard P, Basser PJ, Barnett A, Di Chiro G. Diffusion tensor MR imaging of the human brain. Radiology 1996;201:637-648. 
55. Damoiseaux JS, Smith SM, Witter MP, Sanz-Arigita EJ, Barkhof F, Scheltens $\mathrm{P}$, et al. White matter tract integrity in aging and Alzheimer's disease. Hum Brain Mapp 2008;30:1051-1059.

56. Mufson EJ, Pandya DN. Some observations on the course and composition of the cingulum bundle in the rhesus monkey. J Comp Neurol 2004;225:31-43.

57. Xie S, Xiao JX, Gong GL, Zang YF, Wang YH, Wu HK, et al. Voxelbased detection of white matter abnormalities in mild Alzheimer disease. Neurology 2006;66:1845-1849.

58. Liu M, Gross DW, Wheatley BM, Concha L, Beaulieu C. The acute phase of Wallerian degeneration: longitudinal diffusion tensor imaging of the fornix following temporal lobe surgery. Neuroimage 2013;74: 128-139.

59. Stricker NH, Schweinsburg BC, Delano-Wood L, Wierenga CE, Bangen KJ, Haaland KY, et al. Decreased white matter integrity in late-myelinating fiber pathways in Alzheimer's disease supports retrogenesis. Neuroimage 2009;45:10-16.

60. Rogers H, Arango Lasprilla JC. Retrogenesis theory in Alzheimer's dis- ease: evidence and clinical implications. Anales de Psicología 2006;22: 260-266.

61. Gazzaniga MS. Cerebral specialization and interhemispheric communication Does the corpus callosum enable the human condition? Brain 2000;123:1293-1326.

62. Cloutman LL, Binney RJ, Drakesmith M, Parker GJ, Lambon Ralph MA. The variation of function across the human insula mirrors its patterns of structural connectivity: evidence from in vivo probabilistic tractography. Neuroimage 2012;59:3514-3521.

63. Fleischman DA, Yu L, Arfanakis K, Han SD, Bames LL, Arvanitakis Z, et al. Faster cognitive decline in the years prior to MR imaging is associated with smaller hippocampal volumes in cognitively healthy older persons. Front Aging Neurosci 2013;5:21

64. Chu C, Hsu AL, Chou KH, Bandettini P, Lin C; Alzheimer's Disease Neuroimaging Initiative. Does feature selection improve classification accuracy? Impact of sample size and feature selection on classification using anatomical magnetic resonance images. Neuroimage 2012;60:5970. 